\title{
Academia da Saúde, Academia da Cidade e Academia ao Ar Livre nas agendas política e governamental dos estados brasileiros
}

\author{
Health gym, city gym and outdoor gym on the political and governmental agendas of \\ Brazilian states
}

\section{AUTORES \\ Temistocles Damasceno Silva ${ }^{1}$ (D) \\ Saú da Silva Souza ${ }^{1}$ (iD \\ Fernando Augusto Starepravo 2 (D) \\ 1 Universidade Estadual do Sudoeste da Bahia Departamento de Saúde I, Jequié, Bahia, Brasil. \\ 2 Universidade Estadual de Maringá, Departamento de Educação Física, Maringá, Paraná, Brasil.}

\section{CONTATO}

Temistocles Damasceno Silva

tom@uesb.edu.br

Rua Riachuelo, n. 247, Centro, Jequié,

Bahia, Brasil.

CEP: 45200-030.

DOI

$10.12820 /$ rbafs. $25 \mathrm{e} 0163$

\section{(cc) BY}

Este trabalho está licenciado com uma Licença Creative Commons - Atribuição 4.0 Internacional.

\begin{abstract}
RESUMO
A pesquisa teve como objetivo analisar a atenção dada aos programas Academia da Saúde, Academia da Cidade e Academia ao Ar Livre nas agendas política e governamental das unidades federativas brasileiras. Trata-se de uma pesquisa documental de caráter exploratório. Para organização dos dados, elencou-se três categorias analíticas: o quantitativo de pautas por partido político; a distribuição geográfica das pautas por unidade federativa e região; a prioridades elencadas pelos partidos políticos em relação aos programas abordados. O Partido dos Trabalhadores $(\mathrm{PT})$ do Ceará destacou-se pelo quantitativo de pautas correlatas ao tema investigado. Por outro lado, o Partido Social Cristão (PSC) do Rio de Janeiro propôs o maior número de construção de Academias ao Ar Livre. A maioria das pautas concentraram-se na região Nordeste e foram voltadas, prioritariamente, à oferta de espaços e equipamentos para prática regular de atividade física. Por fim, pode-se concluir que a atenção dada aos programas supracitados nas agendas analisadas se apresenta de maneira embrionária.
\end{abstract}

Palavras-chave: Política pública; Promoção da saúde; Agenda de prioridades em saúde.

ABSTRACT

The research aimed to analyze the attention given to the Health Gym, City Gym and Outdoor Gym programs in the political and governmental agendas of Brazilian federative units. It is an exploratory documentary research. To organize the data, three analytical categories were listed: the number of agendas per political party; the geographical distribution of the guidelines by federative unit and region; the priorities listed by the political parties in relation to the programs covered. The Workers' Party (PT) of Ceara stood out for the number of guidelines related to the subject investigated. On the other hand, the Social Christian Party (PSC) of Rio de Janeiro proposed the largest number of Outdoor Gym to be built. Most of the guidelines were concentrated in the Northeast region and were mainly focused on offering spaces and equipment for regular physical activity. Finally, it can be concluded that the attention given to the programs mentioned in the analyzed agendas is presented in an embryonic way.

Keywords: Public policy; Health promotion; Health priorities agenda.

\section{Introdução}

Em 2006, a criação da Política Nacional de Promoção da Saúde (PNPS) estabeleceu enquanto prerrogativa estatal, o fomento à qualidade de vida e redução dos riscos à saúde da população brasileira. Desta forma, delineou-se enquanto competência dos estados brasileiros, o desenvolvimento de ações intersetoriais relacionadas a promoção da saúde bem como parcerias governamentais para a implantação de atividades relacionadas a política em questão ${ }^{1}$.

Anteriormente, as ações de ordem pública relacionadas à promoção da saúde estavam vinculadas ao Programa Academia da Cidade (PAC), criado em 2002. Tal programa teve como base várias experiências mu- nicipais exitosas no âmbito da promoção da saúde. Esse programa foi implementado em diversas capitais brasileiras através de polos compostos por espaços físicos, equipamentos e recursos humanos voltados a prática regular de atividade física ${ }^{2}$.

Após a promulgação da PNPS surgiram diversas ações no contexto municipal voltadas a promoção da saúde. Dentre elas, destaca-se a iniciativa do professor Roberto Nagahama. Em 2008, o professor atuou como secretário de esporte do município de Maringá, Paraná e instalou aparelhos de ginástica nos espaços públicos voltados a prática regular de atividade física. Com isso, inaugurou-se o Programa Academia ao Ar Livre (AAL) com forte adesão de outros munícipios 
brasileiros ao longo do tempo ${ }^{3}$.

Outro desdobramento da PNPS relaciona-se a criação do Programa Academia da Saúde (PAS) no ano de 2011, alicerçado na premissa do autocuidado através da prática regular de atividade física por meio da oferta de estrutura específica. Tal programa incorporou o PAC enquanto diretriz das ações a serem desenvolvidas ${ }^{2}$. Em 2017, o PAS encontrava-se presente em aproximadamente 48\% dos municípios brasileiros, sendo que, 2.678 municípios foram contemplados com pelo menos um polo do Programa. Nesse montante, $77 \%$ foram municípios de pequeno porte, com menos de 30 mil habitantes ${ }^{5}$.

Apesar desses programas ampliarem o espaço na restrita agenda governamental brasileira poucos estudos retratam a temática ora abordada. Na literatura científica nacional existem análises sobre os atores e processos organizacionais relacionados a construção da PNPS. Além disso, constata-se estudos acerca do processo de constituição da agenda política e as finalidades elencadas ao Estado para a promoção da saú$\mathrm{de}^{6}$. No que se refere ao PAS e PAC, existem estudos sobre a análise dos benefícios desses programas para a saúde da população bem como investigações acerca da redução de iniquidades sociais ${ }^{7}$ e alterações de hábitos alimentares ${ }^{8}$.

Diante do exposto, entende-se o PAC, PAS e a AAL enquanto políticas de saúde que podem se constituir referências empíricas a serem exploradas pelos pesquisadores brasileiros. Uma das possibilidades de abordagem científica desses fenômenos se dá através da compreensão se a atenção dada aos programas em questão manifesta-se de maneira incremental ou sofre rupturas com base nas representações político-partidárias e os seus respectivos atores políticos, refletindo assim, uma mudança na lógica estrutural que determina as prioridades para a política setorial abordada.

Logo, o objetivo da pesquisa foi analisar como os programas Academia da Saúde, Academia da Cidade e Academia ao Ar Livre foram considerados na agenda política e governamental da área setorial da saúde nas unidades federativas brasileiras, no período de 2018 a 2020.

\section{Métodos}

Trata-se de uma pesquisa documental e exploratória com abordagem quanti-qualitativa ${ }^{9}$. Os dados foram analisados com o auxílio do software Nvivo versão 12, programa voltado à análise de informação qualitativa que agrupa ferramentas para o trabalho com do- cumentos textuais, otimizando assim, a organização, a categorização e análise dos dados. O Nvivo possibilitou a codificação de dados similares por meio de unidade de registros denominadas codes. Ao mesmo tempo, foi possível estabelecer relação dessas unidades de registros com os cases (criação de casos para correlação de dados).

Neste contexto, coletou-se os planos de governo (PG) publicados pelas representações político-partidárias dos candidatos eleitos ao cargo de governador das unidades federativas brasileiras durante o processo eleitoral de 2018. Tais documentos encontram-se disponíveis no link DivulgaCand do site do Tribunal Superior Eleitoral. Além disso, coletou-se os Planos Plurianuais (PPA) dos agentes políticos supracitados, disponíveis nos sites das Secretarias Estaduais de Planejamento a partir de 2019. Logo, formou-se um banco de dados para o desenvolvimento da pesquisa. Vale ressaltar que o banco de dados encontra-se disponível no arquivo do Centro de Estudos em Política e Gestão do Esporte e Lazer da Universidade Estadual do Sudoeste da Bahia (CEGEL-UESB).

A escolha dos documentos analisados perpassa pela representatividade dos mesmos enquanto etapas constituintes do processo de formação da agenda política e governamental. O PG apresenta a plataforma de alternativas para os diversos problemas públicos percebidos pelos atores políticos durante o processo eleitoral ${ }^{10}$. O PPA caracteriza-se enquanto um instrumento de planejamento de médio prazo que deve apresentar as prioridades governamentais, conforme as diretrizes do art. 165 da Constituição Federal de 1988.

$\mathrm{Na}$ sequência, os documentos foram exportados para o Nvivo no sentido de organizar e classificar os dados coletados com base na codificação estabelecida. Para tal, elencou-se o termo "academia", incluindo-se palavras derivadas enumeradas pelo software durante a coleta. Os dados foram codificados por meio dos seguintes codes: Academia da Saúde, Academia da Cidade e Academia ao Ar Livre. Paralelamente, os partidos políticos identificados no levantamento preliminar dos documentos supracitados foram transformados em cases na perspectiva de relacioná-los com os codes elencados na pesquisa.

Diante do exposto, o corpus da pesquisa foi constituído por 13 propostas oriundas do PG e 126 propostas do PPA, correlatas aos codes supracitados. Com base nos critérios de inclusão foram mantidas 4 propostas do PG e 78 propostas do PPA. Tais critérios referem-se às propostas vinculadas à academia enquan- 
to variável de promoção da saúde e qualidade de vida, tais como: Academia da Saúde; Academia da Cidade; Academia ao Ar Livre; Academia da Terceira Idade e Academia nas Praças.

Por outro lado, foram excluídas 9 propostas do PG e 48 propostas do PPA. Os critérios de exclusão encontram-se relacionados às propostas que apresentaram distanciamento do objeto de estudo, a saber: academia de letras; academia de polícia; academia das forças armadas; academia enquanto Instituição de Ensino Superior.

Após a codificação dos dados, vinculou-se os codes a cada case. Nesta lógica, foi possível analisar o quantitativo de propostas por partido político, a distribuição geográfica das propostas por Unidade Federativa e região bem como a análise das pautas elencadas com base nos programas analisados. No primeiro momento, examinou-se os dados codificados (codes) em relação aos partidos políticos (cases). Em seguida, analisou-se a distribuição geográfica das propostas por Unidade Federativa (26 Estados e um Distrito Federal) e região (Norte, Nordeste, Centro-oeste, Sudeste e Sul). Para tal, utilizou-se a estatística descritiva no sentido de identificar a frequência das pautas por partido político, unidade federativa e região. Logo, optou-se pela análise de todos os planos de governo e planos plurianuais dos candidatos (as) eleitos (as) para o cargo de chefe do poder executivo estadual durante o processo eleitoral de 2018.

Por fim, investigou-se as pautas identificadas nos PGs e PPAs. A análise ora apresentada encontra alicerce teórico nos pressupostos da agenda-setting delineados por Baumgartner e Jones ${ }^{11}$, acerca da atenção dada a determinada questão na agenda política e governamental. Os autores compreendem as agendas política e governamental enquanto espaços de disputa de poder acerca da atenção dada a determinado tema no processo de tomada de decisão política, levando-se em consideração os diversos fatores que influenciam esse processo, tais como: as ideias, os atores e os arranjos institucionais.

\section{Resultados}

$\mathrm{Na}$ agenda política foram identificadas 4 pautas distribuídas nos PG dos seguintes partidos políticos: Movimento Democrático Brasileiro do Pará (MDB-PA); Partido Democrático Trabalhista do Amapá (PDT-AP); Partido Social Cristão do Rio de Janeiro (PSC-RJ) e o Partido dos Trabalhadores do Rio Grande do Norte (PT-RN). A frequência de propostas por partido político, unidade federativa e região podem ser observadas no Quadro 1. Neste contexto, verificou-se uma homogeneidade dos partidos políticos no que se refere ao número de propostas $(n=1)$ e frequência de pautas por unidade federativa $(25 \%)$.

$\mathrm{Na}$ agenda governamental foram diagnosticadas 78 pautas elencadas por 5 partidos políticos: Movimento Democrático Brasileiro do Distrito Federal (MDB-DF); Partido Socialista Brasileiro de Pernambuco (PSB-PE); Partido Social Cristão do Rio de Janeiro (PSC-RJ); Partido Social Liberal de Roraima (PSL-RR); Partido dos Trabalhadores dos estados da Bahia, Ceará e Piauí (PT-BA, PT-CE, PT-PI). A frequência de propostas por partido político, unidade federativa e região podem ser observadas no Quadro 2. Nesta perspectiva, o PT-CE se apresenta como o partido político com o maior quantitativo de propostas para os programas investigados, correspondendo a 90,41\% $(\mathrm{n}=66)$ da frequência de pautas na região Nordeste. O PT-BA

Quadro 1 - Frequência de pautas para o PAS, PAC e AAL nos Planos de Governo - eleições 2018

\begin{tabular}{|lcc|}
\hline $\begin{array}{l}\text { Unidade Federativa } \\
\text { (UF) }\end{array}$ & $\begin{array}{c}\text { Frequência } \\
\text { Por UF / \% região }\end{array}$ & $\begin{array}{c}\text { Frequência } \\
\text { Por região / \% total }\end{array}$ \\
\hline \multicolumn{3}{|c|}{ Região Norte } \\
\hline Amapá & $1(50 \%)$ & $2(50 \%)$ \\
Pará & $1(50 \%)$ & $1(25 \%)$ \\
\hline \multicolumn{3}{|c|}{ Região Nordeste } \\
\hline Rio Grande do Norte & $1(100 \%)$ \\
\hline \multicolumn{3}{|c|}{ Região Sudeste } \\
\hline Rio de Janeiro & $1(100 \%)$ & $1(25 \%)$ \\
Total & $4(100 \%)$ & $4(100 \%)$ \\
\hline
\end{tabular}

PAS = Programa Academia da Saúde PAC = Programa Academia da Cidade; $\mathrm{AAL}=$ Programa Academia ao Ar Livre.

Quadro 2 - Frequência de pautas para o PAS, PAC e AAL nos Planos Plurianuais (2019 - 2021)

\begin{tabular}{|lcc|}
\hline $\begin{array}{l}\text { Unidade Federativa } \\
\text { (UF) }\end{array}$ & $\begin{array}{c}\text { Frequência } \\
\text { Por UF / \% Região }\end{array}$ & $\begin{array}{c}\text { Frequência } \\
\text { Por Região / \% Total }\end{array}$ \\
\hline \multicolumn{3}{|c|}{ Região Norte } \\
\hline Roraima & $1(100 \%)$ & $1(1,26 \%)$ \\
\hline \multicolumn{3}{|c|}{ Região Nordeste } \\
\hline Bahia & $1(1,36 \%)$ \\
Ceará & $66(90,41 \%)$ & $73(93,58 \%)$ \\
Pernambuco & $4(5,47 \%)$ \\
Piauí & $2(2,73 \%)$ \\
\hline \multicolumn{3}{|c|}{ Região Centro-oeste } \\
\hline Distrito Federal & $2(100 \%)$ \\
\hline \multicolumn{3}{|c|}{$2(2,53 \%)$} \\
\hline Rio de Janeiro & Região Sudeste \\
Total & $2(100 \%)$ & $2(2,53 \%)$ \\
\hline
\end{tabular}

PAS = Programa Academia da Saúde PAC = Programa Academia da Cidade; $\mathrm{AAL}=$ Programa Academia ao Ar Livre. 
apresentou o menor percentual na referida região com $1,36 \%(n=1)$ de frequência das pautas.

Quanto à distribuição geográfica das propostas com base na agenda política, apurou-se a existência de propostas correlatas a AAL em 4 PG de unidades federativas distintas (Figura 1 ). Consequentemente, constatou-se uma concentração de propostas nas regiões Norte, representando assim, 50\% $(\mathrm{n}=2)$ do total de pautas analisadas. Nas regiões Nordeste identificou-se uma pauta no Rio Grande do Norte. Na região Sudeste verificou-se uma pauta no Rio de Janeiro. A soma da frequência de pautas nessas regiões correspondeu a $50 \%$ do total de pautas analisadas.

Em relação a distribuição geográfica na agenda governamental foi possível verificar pautas em 7 unidades federativas (Figura 2). A região Nordeste apresentou uma elevada concentração de pautas $(n=74)$, distribuídas nas seguintes Unidades Federativas: Alagoas ( $\mathrm{n}=$

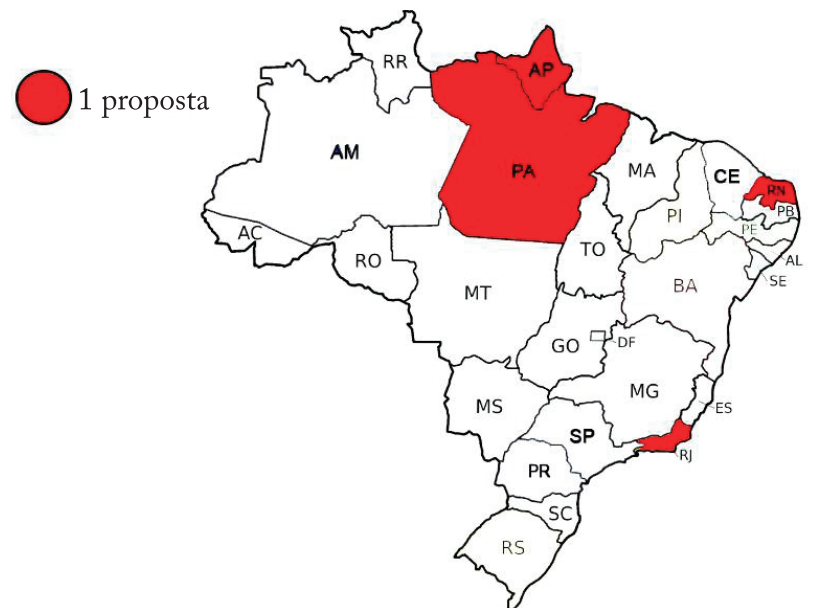

Figura 1 - Distribuição geográfica das pautas para o PAC, PAS e AAL nos PGs



Figura 2 - Distribuição geográfica das pautas para o PAC, PAS e AAL nos PPAs
1), Bahia ( $n=1)$, Ceará $(n=66)$, Pernambuco $(n=4)$ e Piauí $(n=2)$. A referida região correspondeu a $93,6 \%$ do quantitativo total. A região Centro-oeste e a região $\mathrm{Su}-$ deste representaram 2,5\% $(n=2)$, cada. A região Norte representou $1,3 \%(n=1)$ das propostas analisadas.

No que se refere as pautas para AAL elencadas na agenda política diagnosticou-se a realização de parcerias para a garantia de espaços e equipamentos voltados a prática regular de atividade física para a população idosa - MDB-PA, PT-RN e PSC-RJ. Entretanto, tais planos não evidenciaram o quantitativo de espaços a serem construídos. A única proposta distinta diz respeito ao PDT-AP que indicou a criação de uma academia para a prática de atividade física exclusiva aos atletas locais.

$\mathrm{Na}$ agenda governamental verificou-se que o PT-CE priorizou a implantação de 66 academias ao ar livre em diversos municípios do Estado. Além disso, o PT-PI sinalizou a construção de 18 academias similares. O PSL-RR apontou ações voltadas à criação de academias de musculação sem quantificar o número de unidades a serem implantadas. O PSC-RJ designou a criação de 12 unidades da academia do corpo enquanto prioridades do poder público local.

No que diz respeito ao PAC, o PSB-PE defendeu pautas de ampliação da cobertura da rede de Academias da Cidade com a finalidade de requalificação de 4 espaços públicos na perspectiva de contribuir para a humanização desses ambientes por meio da promoção da saúde e do estímulo à prática de atividades físicas. Em relação às propostas voltadas ao PAS, identificou-se que o PT-BA e o MDB-DF listaram propostas de implantação da Academia da Saúde mas não especificaram o quantitativo de polos ou unidades. Apesar de apresentar apenas duas pautas correlatas aos programas analisados, o PSC-RJ destacou-se ao apresentar uma proposta voltada a implantação de 100 unidades da Academia da Saúde.

\section{Discussão}

A atenção dada ao PAC, PAS e AAL nas agendas política e governamental se apresenta de maneira embrionária, levando-se em consideração as diretrizes da Política Nacional de Promoção da Saúde que estabelecem o papel fundamental das unidades federativas brasileiras no desenvolvimento da política em questão.

O reduzido número de pautas nos planos de governo dos partidos políticos analisados corresponde a implantação de AAL voltadas ao público idoso. Após o processo eleitoral percebeu-se uma alternância de prioridades 
em relação ao desenvolvimento das pautas elencadas na agenda política. Apenas o PSC-RJ manteve a promessa de campanha, incluindo assim, a AAL como alternativa para a promoção da saúde da população carioca.

Contudo, tornou-se perceptível a inserção de pautas relacionadas ao PAC, PAS e AAL, nos planos plurianuais de partidos políticos que não propuseram ações para a promoção da saúde durante o período eleitoral analisado. Tais pautas relacionam-se a implantação de infraestrutura para promoção da saúde por meio da criação de Academias da Saúde e Academias da Cidade. Neste contexto, percebe-se a dissonância entre as pautas elencadas na arena política e as prioridades delineadas na agenda governamental.

Logo, verifica-se que os partidos políticos não se apresentam como uma variável explicativa acerca da atenção dada a promoção da saúde. Vale destacar a presença de pautas similares em representações político-partidárias distintas. Um exemplo a ser abordado refere-se às pautas apontadas nos planos plurianuais do Partido dos Trabalhadores e o Partido Social Liberal. De acordo com Tarouco e Madeira ${ }^{10}$, o conteúdo programático dos partidos políticos reflete a imagem que tais entidades desejam expor aos seus filiados, eleitores em potencial e adversários políticos. Por outro lado, o processo de escolha das prioridades para uma determinada política relaciona-se com as coalizões de governo e estratégias políticas.

As pautas concentraram-se na região Nordeste, corroborando assim, com os achados de Gerigk, Ribeiro \& Lepchak ${ }^{12}$, os quais afirmam que os pleitos eleitorais exercem influência nos direcionamentos de recursos públicos para a saúde no contexto municipal da região Nordeste. Basicamente, essas pautas encontram-se vinculadas a construção de espaços e equipamentos para a prática de atividade física por meio da implantação dos programas abordados.

Tais indícios apontam a restrita percepção dos problemas e definição das alternativas para a promoção da saúde no que se refere aos gestores públicos. Além disso, evidencia-se as fragilidades no processo de articulação institucional e as limitações técnico-administrativas relacionadas a formação da agenda governamental para a promoção da saúde, endossando os pressupostos delineados por Fonseca \& Medina ${ }^{1}$.

As limitações da pesquisa referem-se a falta de sistematização dos planos de governo e planos plurianuais, fato esse, prejudicial a análise documental. Além disso, considera-se a dificuldade de compreensão dos dados coletados ao levar em conta o grau de abstração das pautas delineadas pelos partidos políticos. Contudo, acredita-se que os dados da pesquisa possam colaborar com a diagnose do processo de formação da agenda com base na utilização de instrumentos de política voltados a promoção da saúde, neste caso, os programas supracitados. Desta forma, espera-se que os gestores públicos possam utilizar evidências científicas para a qualificação do processo de tomada de decisão política.

\section{Conflito de interesse}

Os autores declaram não haver conflito de interesse.

\section{Financiamento}

O presente trabalho foi realizado com apoio da Universidade Estadual do Sudoeste da Bahia.

\section{Contribuição dos autores}

Silva TD dedicou-se a concepção, desenho do trabalho, coleta de dados e redação do manuscrito. Souza SS participou da coleta de dados e redação do manuscrito. Starepravo FA participou da construção do desenho do trabalho, revisão dos dados e manuscrito. Todos os autores aprovaram a versão final do artigo e colaboraram com a adequação as normas estabelecidas pelo presente periódico.

\section{Agradecimentos}

Os autores agradecem ao Centro de Estudos em Política e Gestão do Esporte e Lazer pelo auxílio na elaboração do banco de dados da pesquisa.

\section{Referências}

1. Fonseca ACF, Medina MG. Promoção da saúde na agenda governamental do estado da Bahia: política ou retórica? Saúde debate. 2018;42(2):302-16.

2. Costa BSL, Mendonça RD, Santos LC, Peixoto SV, Alves M, Lopes ACS. Academia da Cidade: um serviço de promoção da saúde na rede assistencial do Sistema Único de Saúde. Ciênc Saúde Colet. 2013;18(1):95-102.

3. Hammaschimt S, Portela BS. A utilização das academias ao ar livre como promoção da qualidade de vida dos adolescentes. In: Os desafios da escola pública paranaense na perspectiva do professor PDE: produções didático-pedagógicas. Paraná: Governo do Estado, 2014.

4. Malta DC, Castro AM, Gosch CS, Cruz D, Bressan A, Nogueira JD, et al. A Política Nacional de Promoção da Saúde e a agenda da atividade física no contexto do SUS. Epidemiol Serv Saúde. 2009;18(1):79-86.

5. Sá GBAR, Mielke GCDGI, Cruz KG, Ferreira KRD, Silva MMA, Souza MFM, et al. Monitoramento do programa academia da saúde. In: Malta DC, Mielke GI, Costa NCP (eds). Pesquisas de avaliação do programa academia da saúde. 1 ed. Florianópolis: Sociedade Brasileira de Atividade Física e Saúde, 2020, p.196-216. 
6. Mannheimer LN, Lehto J, Östlin P.Window of op- portunity for intersectoral health policy in Sweden-open, half-open or half-shut? Health Promot Int. 2007;22(4):307-15.

7. Carvalho SR. Saúde coletiva e promoção da saúde: sujeito e mudança. 2a ed. São Paulo: Hucitec, 2007.

8. Heidmann ITS, Buss AMCP, Boehs AE, Wosny AM, Monticelli M. Promoção à saúde: trajetória histórica de suas concepções. Texto Contexto Enferm. 2006;15(2):352-358.

9. Gil AC. Métodos e técnicas de pesquisa social. $6^{\mathrm{a}}$ ed. São Paulo: Atlas, 2008.
10. Madeira RM, Tarouco GS. Esquerda e Direita no Brasil: uma análise conceitual. Rev Cien Soc. 2011;8(15):1-25.

11. Baumgartner FR, Jones, BD. Agendas and Instability in American Politics. Chicago: University of Chicago Press, 1993.

12. Gerigk W, Ribeiro F, Lepchak A. Desempenho fiscal dos pequenos municípios brasileiros. RC\&C. 2019;11(1):85-103.

Recebido: 01/08/2020

Aprovado: 20/11/2020

\section{Como citar este artigo:}

Silva TD, Souza SS, Starepravo FA. Academia da Saúde, Academia da Cidade e Academia ao Ar Livre nas agendas politica e governamental dos estados brasileiros. Rev Bras Ativ Fís Saúde. 2020;25:e0163. DOI: 10.12820/rbafs.25e0163 